\title{
BLACK HOLES CANNOT SUPPORT CONFORMAL SCALAR HAIR
}

\author{
T. Zannias* \\ Department of Physics \\ Queen's University \\ Kingston, Ontario \\ Canada \\ K7L 3N6
}

\begin{abstract}
It is shown that, the only static asymptotically flat non extrema black hole solution of the Einstein-conformally invariant scalar field equations having the scalar field bounded on the horizon, is the Schwarzschild one.Thus black holes cannot be endowed with conformal scalar hair of finite length.
\end{abstract}

\footnotetext{
* PACS 04.20.Ex,97.60Sm
} 


\section{INTRODUCTION}

To a physicist, knowing the spectrum of asymptotically flat black hole solutions of the Einstein field equations it has been always of a vast interest. The classical black hole uniqueness theorems assert that as far as the Einstein-Maxwells equations are concerned, the only asymptotically flat, black hole equilibrium states are those and only those included in the Kerr-Newman family of metrics. It is not clear however, what the state of affairs is whenever other field configurations are considered as sources of Einstein equations. Further,whereas some time ago relativists had faith in the so called "no hair conjecture", ie in the statement that black holes always have their exterior empty, apart from an electromagnetic field, the conjecture appears now days to faint away. The black hole solution discovered in [1] implies that a dilaton coupled to a Maxwell field can withstand the inward pull of gravity and thus peacefully coexists with the black hole.On the other hand the recently analyzed [2] Einstein non Abelian Yang-Mills equations shows that a non trivial Yang-Mills field is a genuine hair and thus the "colored" class of black holes provides another counterexample against the "no hair conjecture".For the moment however there is no theory telling us which sources admit hairy black holes and which do not.In the absence of such theory one has to consider explicitly various energy- momentum tensors as source of gravity and try to detect the nature of the admitted black holes.

The purpose of the present paper is to look for black holes solutions admitted by the coupled Einstein-conformally invariant scalar field equations. Although it is well known [3] that a minimally coupled to gravity massless scalar field taken as a source of Einsteins gravity admits only the Schwarzschild black hole as a solution,it is not yet known whether the same holds true for the conformally invariant system. On the contrary the conformal system does admit a black hole solution.It discovered by Bekenstein [4] and it shares the following properties: 
1a) It is spherically symmetric and extrema

1b) The scalar field is unbounded on the horizon.

Despite its shortcomings (see [5] but also [4]) the existence of the "Bekensteinblack hole" raises a number of related questions.For instance: Is the Bekenstein black hole unique? Do there exist other black holes solutions of the system where the field obeys different boundary conditions on the horizon? If they do exist,are all of them spherical,or (and) extrema? It is the purpose of the present paper to investigate some of the above questions.More precisely we shall search for black holes solutions which are:

2a) non extrema

$2 \mathrm{~b})$ the conformal scalar field is bounded on the horizon.

It will be shown in the following sections that there is only one class of black holes states satisfying $(2 \mathrm{a}, 2 \mathrm{~b})$. More precisely it will be proven that the only static asymptotically flat black hole solution obeying properties $(2 \mathrm{a}, 2 \mathrm{~b})$ is the Schwarzschild one having vanishing exterior conformally invariant scalar field.The result will be shown in two different but complementary to each other methods. In the first approach we look for black hole states with the property that the non trivial scalar field $\Phi$ is bounded on the horizon ie $\Phi^{2}<\alpha^{-1}$, with $\alpha$ a constant to be determined later on.In this case we shall exploit the local equivalence of the conformal system to the minimal one [6] and this fact will allows us to prove a stronger statement. Namely the absence of multipole static black hole endowed with conformal hair. The second method removes the restriction upon the scalar field.In that sense it is more general than the first one but it restricts the topology of the event horizon (and in that sense the results are not as general as the first). Specificaly it will be assumed that the event horizon consists only of a single connected component. Under this hypothesis we show that any static black 
hole solution must have vanishing scalar hair and thus be Schwarzschild. Although the connectedness assumption of the horizon is likely to be irrelevant, our method of establishing the proof needs it.

We shall begin the paper by first giving a new prof of the black hole uniqueness theorem for the minimally coupled system. The proof utilizes a theorem proven by Shoen and Yau [9] and follow arguments similar to those employed by Bunting and ul-Alam [7] and others [8] in re-deriving known black hole uniqueness theorems. As a byproduct we also show that the the Einstein-minimal coupled massless scalar field equations do not admit multipole black hole in static equilibrium. The main results for the conformal case are derived in section two and three and we finish the paper with a discussion related to the uniqueness of the Bekenstein black hole.

\section{Uniqueness Theorem for the minimal system.}

Some time ago, Chase and Bekenstein [3] established the following theorem:The only static, asymptotically flat, black hole solution of the Einstein-minimally coupled scalar field equations is the Schwarzschild black hole possessing a trivial (ie vanishing) exterior field. We shall present a new prof of the above theorem the results of which, will serve as an intermideate step in reaching our goal for the conformal coupling.

We begin by introducing a set of coordinates suitable for descriding static black hole exterio. The presence of the hypersurface orthogonal Killing field allows us to introduce coordinates so that the line element in that part of the spacetime reads:

$$
d s^{2}=-V^{2} d t^{2}+g
$$

$V^{2}$ stands for the square of the timelike Killing field and $g$ is a Riemmanian metric on the $t=$ Const spaces. In the above set of coordinates, the coupled Einstein-minimal 
scalar field equations are equivalent to:

$$
\begin{gathered}
R_{\alpha \beta}=k D_{\alpha} \Phi D_{\beta} \Phi+V^{-1} D_{\alpha} D_{\beta} V \\
D^{\alpha} D_{\alpha} V=0 \\
D^{\alpha} D_{\alpha} \Phi=-V^{-1} D^{\alpha} V D_{\alpha} \Phi
\end{gathered}
$$

where the Ricci, all covariant derivatives,raising and lowering indices are in terms of the positive definite metric of $t=$ const slice.Any $t=$ const slice shall be refered here after by $\Sigma$, while the gravitational coupling constant is denoted by k. The three metric $g$ and the field $\Phi$ are assumed to exhibit the familiar fall of rate at the asymptotic region of $\Sigma$ ie:

$$
\begin{gathered}
g_{\alpha \beta}=\left(1+2 m r^{-1}\right) \delta_{\alpha \beta}+h_{\alpha \beta} \\
V=1-2 m r^{-1}+u \\
\Phi=e r^{-1}+f
\end{gathered}
$$

where the functions $\mathrm{h}, \mathrm{u}$, and $\mathrm{f}$ are assumed to be $O\left(r^{-2}\right)$ possessing $O\left(r^{-3}\right)$ derivatives. On the other hand the internal boundary $b d \Sigma$ of $\Sigma$ defined by $V=0$ will de assumed to be union of regular closed two spaces on which the four dimensional scalar $K=$ $R^{M N K A} R_{M N K A}$ should be bounded. It is appropriate at this point to recall the ShoenYau theorem [9].It states that an asympotically flat,complete, orientable Riemannian manifold $(M, \gamma)$ possesing zero ADM mass,and non negative scalar curvature,must be flat.This conclusion constitutes the backbone for new elegant proofs of various black hole uniqueness theorems $[7,8]$. The idea behind the new proofs is to consider first two copies of $\Sigma$ say $\Sigma_{-}, \Sigma_{+}$and introduce Riemannian metrics $g_{-}, g_{+}$on $\Sigma_{-}$and $\Sigma_{+}$respectively. The first one posseses zero ADM mass, as measured from the asymptotic region of $\Sigma_{+}$, while $g_{-}$exhibits asymptotic properties guaranteeing that if one add a point $P$, standing for the infinity of $\Sigma_{-}$then, $M=\Sigma_{-} U\left(b d \Sigma_{ \pm}\right) U \Sigma_{+}$is a complete,non singular without boundary Riemannian manifold. The authors of [7] construct the metrics $g_{ \pm}$ 
by conformally transforming the physical metric $g$ of $\Sigma$. By an appropriate choice of the conformal factor $\Omega$ one may be able to meet all the conditions required by the ShoenYau theorem. For our problem we choose $\Omega_{ \pm}=\frac{1}{4}(1 \pm V)^{2}$ and consider $g_{ \pm}=\Omega_{ \pm}^{2} g$, as the two Riemannian metrics on $\Sigma_{ \pm}$respectively.By construction the ADM mass of $g_{+}$is zero,and the asymptotic behaviour of $V, g$, implies that by additing a point $P$ ,$g_{-}$compactifies the infinity of $\Sigma_{-}$.(For more details about the construction the reader may consult ref[7].) Furter,one may easily prove that $g_{ \pm}$join smoothly along the two "spheres" defined by $V=0$. and the scalar curvature of $g_{ \pm}$reads:

$$
R\left[g_{ \pm}\right]=k \Omega^{-2} D^{\alpha} \Phi D_{\alpha} \Phi
$$

which manifestly is not negative. Thus according to the Shoen-Yau theorem $g_{ \pm}$must be flat which in turn implies (according to the above formula) that the gradient of $\Phi$ must be zero. Therefore the scalar field is constant over $\Sigma_{+}$and because it vanishes at infinity it must vanish everywhere. To complete the proof and thus show that the physical metric $g$ is spherical and more precisely Schwarzschild one uses the conformally flat nature of $g$ and appeals to the familiar arguments [10]. (Although we have never mentioned it explicitly, it might be worth keeping in mind that the proof requires the gradient of $\Phi$ to be bounded on the horizon. Otherwise the curvature of $g_{ \pm}$will be singular and thus one cannot appeal to Shoen-Yau theorem.)

\section{The conformal system with $\Phi^{2}<\alpha^{-1}$}

Let us now move to our main problem ie the examination of the coupled EinsteinConformally invariant scalar field equations. The theory is described by the following set of equations:

$$
\begin{gathered}
R_{M N}=\left(\frac{\alpha}{1-\alpha \Phi^{2}}\right)\left[4 \nabla_{M} \Phi \nabla_{N} \Phi-2 \Phi \nabla_{M} \nabla_{N} \Phi-g_{M N} \nabla^{\Gamma} \Phi \nabla_{\Gamma} \Phi\right] \\
\nabla^{A} \nabla_{A} \Phi=0
\end{gathered}
$$


where all indices are four dimensional and $\alpha=k 6^{-1}$. The system is much more complicated than say the minimally coupled one. The "conformal" stress tensor contains second derivatives of the scalar field and this fact introduces additional complications demanding special care. Even looking for exact solutions is a rather difficult and painful job [11]. Our task is to investigate whether the coupled system admits asymptotically flat black hole states obeying conditions $(2 \mathrm{a}, 2 \mathrm{~d})$. Let us assume that it does so.At first note that the non extrema property of the horizon implies non vanishing surface gravity and the $t=$ cons slices smoothly intersect the bifurcation two spheres [12]. Projecting the above equations along the $t=$ cost spaces one get an equivalent set:

$$
\begin{gathered}
R_{\alpha \beta}=\alpha \Lambda\left[4 D_{\alpha} \Phi D_{\beta} \Phi-2 \Phi D_{\alpha} D_{\beta} \Phi-g_{\alpha \beta} D^{\gamma} \Phi D_{\gamma} \Phi\right]+V^{-1} D_{\alpha} D_{\beta} V \\
V^{-1} D^{\alpha} D_{\alpha} V=\alpha \Lambda\left[D^{\alpha} \Phi D_{\alpha} \Phi+2 \Phi V^{-1} D^{\alpha} \Phi D_{\alpha} V\right] \\
D^{\alpha} D_{\alpha} \Phi=-V^{-1} D^{\alpha} \Phi D_{\alpha} V
\end{gathered}
$$

where we denote here after:

$$
\Lambda=\frac{1}{1-\alpha \Phi^{2}}
$$

The coupled equations can be transformed to a set describing a self gravitating minimally coupled scalar field.Such a reduction has been known for some time $[6,13]$ and it has been used as a mechanism to generate solutions of the conformal system from that of the minimal one. The reduction can be accomplished via a conformal transformation of the metric and simultaneous field redefinition. To benefit from this property of the conformal system one must search for a smooth conformal factor which:

3a) should not vanish neither within $\Sigma$ nor on $b d \Sigma$.

3b) should transform the conformal black hole solution to an asumptotically flat solution of the minimal sustem. 
For at least one case such a conformal factor (actually an equivalence class) can be constructed explicitly.Before we do so, let us introduce a new metric via:

$$
\bar{g}=\left(1-\alpha \Phi^{2}\right) g
$$

and simultaneously define a new red shift factor $\bar{V}$ and field $\bar{\Phi}$ by:

$$
\begin{gathered}
\bar{V}=\left(1-\alpha \Phi^{2}\right)^{\frac{1}{2}} V \\
\Phi=\alpha^{-\frac{1}{2}} \tanh \left(\alpha^{\frac{1}{2}} \bar{\Phi}\right.
\end{gathered}
$$

It takes some calculations to show that because of (5a-c) $\bar{g}, \bar{V}$ and $\bar{\Phi}$ obey:

$$
\begin{gathered}
R[\bar{g}]=k \bar{D}^{\alpha} \overline{\Phi D}_{\alpha} \bar{\Phi} \\
\bar{D}^{\alpha} \bar{D}_{\alpha} \bar{V}=0 \\
\bar{D}^{\alpha} \bar{D}_{\alpha} \bar{\Phi}=-\overline{V D}^{\alpha} \overline{\Phi D}_{\alpha} \bar{\Phi}
\end{gathered}
$$

A comparison between the above set of equations and (1a-c) shows that the barred quantities indeed satisfy field equations identical to those obeyed by the minimally coupled system.Therefore any conformally transformed black hole solution is mapped locally to solution of the minimal system. However that by itself allows us to conclude almost nothing. We must demonstrate that the conformal factor $\Omega^{2}=1-\alpha \Phi^{2}$ is a "good" one, ie obeys properties (3a,3b).A-priori however there is no fundamental reason that the above defined $\Omega$ should satisfy them. It is at this point where restrictions upon $\Phi$ will be imposed. Notice that by applying maximum principle to the equation (5c) one concludes that $\Phi$ cannot have neither maximum nor minimum in the interior of $\Sigma$. Thus $\Phi$ is monotonic over $\Sigma$ and consequently if the conformal factor is positive on the horizon it will remain so within the interior of $\Sigma$. This will be the case provided on the horizon [13] the field satisfies $\Phi^{2}<\alpha^{-1}$. In such case it is clear that our choice of the conformal factor globally maps the conformal black hole solution to a coressponding 
black hole of the minimal system. However because the latter system admits only one class of black holes namely the Schwarzschild class,it is clear from (8a) that our initial conformal black hole solution must have vanishing scalar field and further be spherical ie be the Schwarzschild black hole. Notice again, that the proof requires the gradient of $\Phi$ to be finite on the horizon, a point that will be discussed at length latter on.

\section{The case with $\Phi^{2} \geq \alpha^{-1}$}

Although it was relatively easy to rule out the existence of hairy black holes with the property $\Phi^{2}<\alpha^{-1}$ ruling out the existence of black holes obeying $\Phi^{2} \geq \alpha^{-1}$ over the horizon it is not straightforward. This is due to the fact that the obvious cice $\Omega^{2}=\alpha \Phi^{2}-1$ would not any longer do the job.One may prove that the conformal solution is mapped onto a non asymptotically flat solution of the minimal system [6b] a conclusion that leads us to nowhere.We shall circumvented the difficulty by employing an alternative method.Unfortunately however, at some cost. Whereas so far we let the horizon to be arbitrary union of two "spheres" corresponding to the notion of multiple black holes, from now on some restriction will be imposed. In particularly we shall assume that the horizon consists of a single connected component.In turn, this will allows us to use some of the machinery developed by Israel [14] in establishing the uniqueness of the Schwarzshild black hole. Recall in Israels approach one uses $V$ as one of the coordinates and projects the field equation on each $V=$ cons two space. They naturally split into a set of dynamical equations propagating the intrinsic metric $g_{i j}$ extrinsic curvature $K_{i j}$, and fields from one two space into next one and a set of constraints equations relating the dynamical variables on some initial surface.For our purpose we shall explicitly use only the constraint system. An insightful tool for our subsequent analysis is the four dimensional scalar $R^{A B K M} R_{A B K M}$. In Israels approach 
takes the form:

$$
\left.\frac{1}{4} R^{A B K M} R_{A B K M}=G_{\alpha \beta} G^{\alpha \beta}+\frac{K^{i j} K_{i j}}{\rho^{2} V^{2}}+\frac{2 D^{i} \rho D_{i} \rho}{\rho^{4} V^{2}}+\frac{1}{\rho^{2} V^{2}}\left[\frac{k \rho V}{2}\left(\mu+{ }^{3} T\right)+K\right)\right]^{2}
$$

where $G_{\alpha \beta}$ is the three dimensional Einstein tensor, $K$ is the trace of the extrinsic curvature of the $V=$ const equipotential two-spaces and $\rho^{-2}=D \alpha V D_{\alpha} V$. The scalars $\mu,{ }^{(3)} T$ are contractions of the conformal stress tensor and are giving by:

$$
\begin{gathered}
\mu=T_{M N} n^{M} n^{N}=\frac{1}{\left(1-\alpha \Phi^{2}\right)}\left[\frac{2 \Phi}{V} D^{\alpha} V D_{\alpha} \Phi+D^{\alpha} \Phi D_{\alpha} \Phi\right] \\
{ }^{3} T=T_{\alpha \beta} g^{\alpha \beta}=T_{M N} h_{\alpha}{ }^{M} h_{\beta}{ }^{N} g^{\alpha \beta}=\frac{1}{1-\alpha \Phi^{2}}\left[D_{\alpha} \Phi D^{\alpha} \Phi+\frac{2 \Phi}{V} D^{\alpha} \Phi D_{\alpha} V\right]
\end{gathered}
$$

where $n^{A}, h_{\alpha}{ }^{M}$ are the unit normal of the $t=$ const slice and the projection tensor on the slice respectively.There are two particular places where $R^{A B M N} R_{A B M N}$ pics up peculiar contributions due to the conformally invariant nature of $\Phi$. First in the combination $\mu+{ }^{3} T$ and secondly in the three dimensional Einstein tensor where second derivatives of $\Phi$ are appearing.Both of them have to be regular on the horizon. Let $g, V, \Phi$ describes a black hole solution subject $\Phi \geq \alpha^{-\frac{1}{2}}$. (Although this is the case we like to investigate,it ought to be stressed that the following proof is independent upon the particular value of $\Phi$ on the horizon). We shall first show that if $\Phi$ is bounded on the horizon, then there it must be constant with vanishing three gradient.The claim is a consequence of the regularity of $R^{A B M N} R_{A B M N}$ on the horizon. Regularity of the right hand side of (6a) on $V=0$, implies that $V=0$ is a totally geodesic two-space on which $D_{i} \rho=O\left(\rho^{2} V\right)$. Further taking into account (6a,c) one concludes that the combination $\mu+{ }^{3} T$ is finite on the horizon, provided the expression :

$$
\frac{\alpha}{1-\alpha \Phi^{2}} \frac{2 \Phi}{V} D^{\alpha} V D_{\alpha} \Phi
$$

is bounded there.Since extremal horizons have been excluded,one therefore one concludes that the above term makes a regular contribution provided one of the following alternatives holds: 
4a) The field $\Phi$ is unbounded on the horizon.For instance if $\Phi=O\left(V^{-1}\right)$ and $D^{\alpha} \Phi D_{\alpha} V$ is bounded then everything is fine on the horizon [15].

4b) The field $\Phi$ is bounded but $D_{\alpha} \Phi=O(V)$

As we have already discussed in the introduction alternative 4a) has been excluded from further considerations so attention will be will be restricted to the second case. But condition (4b) implies that $\Phi$ is constant over the horizon say $\Phi=\Phi_{o}$. Finally to conclude the regularity of the entire right handside of (9a) over the horizon we outght to examine the second derivatives of $\Phi$ hiden in the three dimensional Einstein tensor (see eqs (5ab)) For points interior to $\Sigma, \Phi$ obeys an elliptic equation and as long as the geometry is not singular one expects $\Phi$ to be smooth and in fact analytic.For horizon points however this argument is not sufficient. We shall show that if (4b) holds, then the second derivatives of $\Phi$ on the horizon are bounded. To prove it, we shall use the constraint system of the Israel formalism. Adopted for our case it has the form:

$$
\begin{gathered}
\frac{1}{2}\left({ }^{2} R+K^{i j} K_{i j}-K^{2}\right)=-\alpha T_{\alpha \beta} n^{\alpha} n^{\beta}+\frac{K}{V \rho} \\
\overline{D_{c}}\left(K_{i}^{c}-\delta^{c}{ }_{i} K\right)=\alpha T_{a i} n^{\alpha}-\frac{1}{\left(V \rho^{2}\right)} \overline{D_{i}} \rho
\end{gathered}
$$

with the components of $T_{\alpha \beta}$ giving by:

$$
T_{\alpha \beta}=\Lambda\left(4 D_{\alpha} \Phi D_{\beta} \Phi-2 \Phi D_{\alpha} D_{\beta} \Phi-g_{\alpha \beta} D^{\gamma} \Phi D_{\gamma} \Phi\right)
$$

The overbarred covariant derivative differentiates tangentially and is formed out of the induced two metric, while the reader is reminded that latin indices are two dimensional. Specializing the above equations to the $V=0$ space and taking into account (4b) one finds that the second constraint is automatically satisfied while the first reduces to:

$$
\frac{1}{2}\left(1-\alpha \Phi^{2}\right)\left({ }^{2} R-\frac{2 K}{V \rho}\right)=2 \alpha \Phi n^{\alpha} n^{\beta} D_{\alpha} D_{\beta} \Phi
$$

The above constraint relates $\Phi$ and its second radial derivative on the horizon to the Gaussian curvature ${ }^{2} R$ and extrinsic curvature. Because the intrinsic geometry is regular 
one concludes that the second radial derivative of $\Phi$ is bounded on the horizon. The tangential and other mixed derivatives are vanishing as a consequence of the constancy of $\Phi$ over the horizon. Notice that as a consequence of (10) one gets even stronger conclusion. The ratio

$$
\frac{D_{\alpha} D_{\beta} \Phi}{1-\alpha \Phi^{2}}
$$

is always bounded on the horizon which also implies regularity even in the case where on the horizon $\Phi^{2}=\alpha^{-1}$.

Having in mind the above detailed boundary behaviour of $\Phi$ on the horizon, let us multiply both sides of (5c) by $V$.It leads to

$$
D^{\alpha}\left(V D_{\alpha} \Phi\right)=0
$$

Multiplying further by $\Phi$, integrating over $\Sigma$ and applying Stokes theorem one arrives at:

$$
\oint V \Phi D_{\alpha} \Phi d \Sigma^{\alpha}=-\int V D^{\alpha} \Phi D_{\alpha} \Phi d^{3} V
$$

where the surface integral is taken over the $V=0$.The contribution from the surface integral at infinity has been neglected since the asymptotic behaviour of the geometry and $\Phi$ implies that the integrand is $O\left(r^{-3}\right)$ at infinity. Taking into account the behaviour of $\Phi, D_{\alpha} \Phi$ on the inner boundary we see that there is not any contribution either. Therefore the left side of above equation is vanishing implying $D_{\alpha} \Phi$ is zero on $\Sigma$ ie $\Phi$ is identically zero in the black hole exterior.Proving further that the system (5a-c) with $\Phi=0$ admits only the Schawrtzschild black hole as a solution is by now well known.

\section{Discussion}

Although we have proven the absence of non trivial static black holes endowed with conformal hair of finite length and possesing non degenerate horizons, the case 
of black holes admitting infinite conformal hair is still open. For the later case, it is of great interest to know whether there exist non extrema solutions. Surely if they do exist they cannot be spherical.This is so because of a limited uniqueness theorem obeyed by the Bekenstein solution. It was shown elsewhere [16] that amongst all static, asymptotically flat, spherical solutions of the system, the only black hole solution is the (extrema) Bekenstein black hole.This result although very useful it is limited, because it only examines the spherically symmetric sector of the system and does not rule out static axisymmetric black hole solutions. This seem to be unlike and all existing evidences suggest that the only non trivial static,asymptotically flat black hole solution of the Einstein- conformally invariant scalar field eqs.is the Bekenstein black hole.At the moment there is no proof of this statement. It may be worth to reminder the reader that nothing is known about existence of stationary black hole states admitted by the conformal system.

This paper is the outcome of an active and enjoyable collaboration of the author with the late B.C.Xanthopoulos and it constitutes a natural extension of the results presented in ref [16].I take the oppurtunity to dedicate it to his memory.

Part of this research was done while the author was a a CITA National Fellow and was partially supported by a grant from the Principals Development Fund of Queens University. 


\section{bf References}

[1] G.W.Gibbons Nucl.Phys. B207,337,(1982)

G.W.Gibbons and K.Maeda Nucl.Phys. B298,741,(1988)

G.Horowitz,A.Strominger,S.Giddings Phys.Rew.D43,3140 (1991)

(Err.D45,3888,1992)

[2] P.Bizon,Phys.Rev.Lett. 64,2844 (1990)

M.S.Volkov and D.V.Galtsov,Sov.J.Nucl.Phys. 51,747 (1990)

H.P.Kunzle and A.K.M.Masood-ul-Alam,J.Math.Phys. 31,928(1990)

See also: D.Sudarsky and R.M.Wald,Phys.Rev.D46,1453 (1992)

[3] J.E.Chase,Commun.Math.Phys. 19,276 (1970)

J.D.Bekenstein,Phys.Rev.D5,2941 (1972)

[4] J.D.Bekenstein Ann.Phys.(N.Y) 82, 535 (1974)

Various properties of the solution are discussed in:

J.D.Bekenstein Ann.Phys.(N.Y.) 91,72. (1975)

[5]The solution however appears to be unstable see for instance:

N.Bocharova,K.Bronnikov and V.Melnikov Vestn.Mosk.Univ.Fiz.Astron.

6,706, (1970)

[6]See J.D.Bekenstein in ref [4], also

B.C.Xanthopoulos and A.DialynasJ.Math.Phys.J.Math.Phys.33,1463,(1992)

[7]G.L.Bunting and A.K.M. Masood-ul-Alam Gen.Rel.Grav.19,174 (1987)

[8] A.K.M Masood-ul-Alam Class.Quantum Grav. 9,L53 (1992)

M.Heusler Class.Quantum Grav. 11 ,L49 (1994)

P.Ruback Class.Quantum Grav. 5 ,L155 (1988)

[9] R. Schoen and S.T. Yau, Commun. Math Phys. 65,45 (1979),also

R.Shoen and S.T.Yau,Commun. Math Phys. 79, 231, (1981),

[10]D.C.Robinson G.R.G 8,695,(1977)

see also L.Lindblom J.Math.Phys.21,1455,(1980) 
[11] B.C. Xanthopoulos and T.Zannias J.Math. Phys. 32,1875 (1991)

B.C. Xanthopoulos and T.Zannias J.Math.Phys. 33, 1462 (1992)

[12]I.Racz anb R.M.Wald Class.Quantum Grav.9,2643,(1992)

[13]It ought to be reminded that the self gravitating coupled system it is not even scale invariant.Thus if $\left(g_{M N}, \Phi\right)$ is a solution of the system one cannot alter the value of $\Phi$ on the horizon by a rescaling of the form $c^{2} g_{M N}, c^{-1} \Phi$ with $c$ an arbitrary constant.The only case one can do that is via rescaling of the form $\left(g_{M N}, c \Phi\right)$ and simultaneously renormalizing the coupling constant to a new value $k c^{-2}$.However in this case the numerical value of $1-\alpha \Phi^{2}$ remains invariant.

[14]W.Israel Phys.Rev 164,1776(1967) and Comm.Math.Phys. 8,245,(1968)

[15] Actually this case requires a rather delicate balance between the way $D^{a} \Phi D_{a} \Phi$ and $\Phi$ approach infinity.

[16]B.C.Xanthopoulos and T.Zannias J.Math.Phys.32,1875,(1991) 